\title{
Multisensory Integration in the Superior Colliculus Requires Synergy among Corticocollicular Inputs
}

\author{
Juan Carlos Alvarado, Terrence R. Stanford, Benjamin A. Rowland, J. William Vaughan, and Barry E. Stein \\ Department of Neurobiology and Anatomy, Wake Forest University School of Medicine, Winston-Salem, North Carolina 27157
}

Influences from the visual (AEV), auditory (FAES), and somatosensory (SIV) divisions of the cat anterior ectosylvian sulcus (AES) play a critical role in rendering superior colliculus (SC) neurons capable of multisensory integration. However, it is not known whether this is accomplished via their independent sensory-specific action or via some cross-modal cooperative action that emerges as a consequence of their convergence on SC neurons. Using visual-auditory SC neurons as a model, we examined how selective and combined deactivation of FAES and AEV affected SC multisensory (visual-auditory) and unisensory (visual-visual) integration capabilities. As noted earlier, multisensory integration yielded SC responses that were significantly greater than those evoked by the most effective individual component stimulus. This multisensory "response enhancement" was more evident when the component stimuli were weakly effective. Conversely, unisensory integration was dominated by the lack of response enhancement. During cryogenic deactivation of FAES and/or AEV, the unisensory responses of SC neurons were only modestly affected; however, their multisensory response enhancement showed a significant downward shift and was eliminated. The shift was similar in magnitude for deactivation of either AES subregion and, in general, only marginally greater when both were deactivated simultaneously. These data reveal that SC multisensory integration is dependent on the cooperative action of distinct subsets of unisensory corticofugal afferents, afferents whose sensory combination matches the multisensory profile of their midbrain target neurons, and whose functional synergy is specific to rendering SC neurons capable of synthesizing information from those particular senses.

\section{Introduction}

Previous studies have shown that superior colliculus (SC) multisensory neurons require inputs from the anterior ectosylvian sulcus (AES) to integrate the information they receive from different senses. In the absence of such cortical inputs, they retain the ability to respond to the different sensory stimuli (i.e., they are still "multisensory"), but their response to a combination of cross-modal stimuli is no longer significantly different from that to the most effective component stimulus (Wallace and Stein, 1994; Jiang et al., 2001; Stein et al., 2004; Alvarado et al., 2007a). In short, being multisensory does not guarantee that the neuron can combine (i.e., integrate) its inputs. The same dependencies of multisensory integration on cortex seen in SC neurons are also seen in SC-mediated orientation behaviors (Wilkinson et al., 1996; Jiang et al., 2002, 2007)

Although little is known about how the AES projection fosters SC multisensory integration, possibilities are suggested by the specificity of its projections. This dense corticocollicular projection (Stein et al., 1982; McHaffie et al., 1988; Meredith and Clemo, 1989; Meredith et al., 1992; Harting et al., 1997) is derived from unisensory neurons in the AES (Wallace et al., 1993), and

\footnotetext{
Received Feb. 1, 2009; revised March 31, 2009; accepted April 5, 2009.

This work was supported by National Institutes of Health Grant EY016716. We thank N. London for editorial assistance.

Correspondence should be addressed to Juan Carlos Alvarado at his present address: Regional Center for Biomedical Research, Department of Medical Sciences, School of Medicine, University of Castilla-La Mancha, $02006 \mathrm{Al}$ bacete, Spain. E-mail: juancarlos.alvarado@uclm.es.

DOI:10.1523/JNEUROSCI.0525-09.2009

Copyright $\odot 2009$ Society for Neuroscience $\quad$ 0270-6474/09/296580-13\$15.00/0
}

has a distinctive pattern. The unisensory efferents from each of the three AES subregions: the anterior ectosylvian visual area (AEV) (Mucke et al., 1982; Olson and Graybiel, 1987; Benedek et al., 1988; Jiang et al., 1994); the auditory field of AES (FAES) (Clarey and Irvine, 1986; Meredith and Clemo, 1989); and the fourth somatosensory area (SIV) (Clemo and Stein, 1982, 1983) are combined in patterns that match the modality-convergence profile of their SC target neurons (Wallace et al., 1993). For example, SC neurons that receive unisensory visual and auditory input from other sources also receive descending inputs from AEV and FAES, but not from SIV.

Evidence that SC multisensory neurons receive converging modality-matched cortical inputs suggests a functional synergy among the inputs from different AES subregions, wherein each unisensory cortical input is necessary, but not sufficient, for normal multisensory integration. The term "synergy" here refers to a nonlinear relationship such that one input alone would be insufficient to maintain integration. Thus, it would only be via their cooperative impact on an SC neuron that this capability for multisensory integration is able to be realized. However, the opposite is also possible. As noted above, prior studies have demonstrated that while AES enables multisensory integration, it does not provide the primary cross-modal drive to SC multisensory neurons. From a computational perspective, the precise role of the AESderived inputs is unclear and there remains the possibility that these unisensory AES inputs are redundant, rather than synergistic. Accordingly, any given AES input would be sufficient to support the integration of the multiple sensory inputs that an SC neuron obtains from other sources. The present study was de- 
A Cooling coil In FAES

\section{Control active}
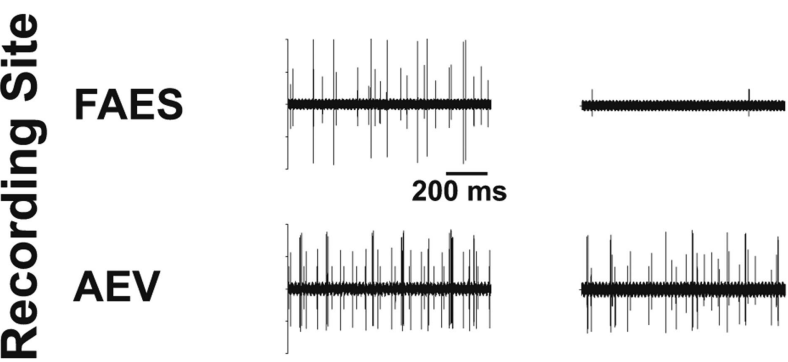

Recovery

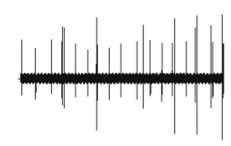

B

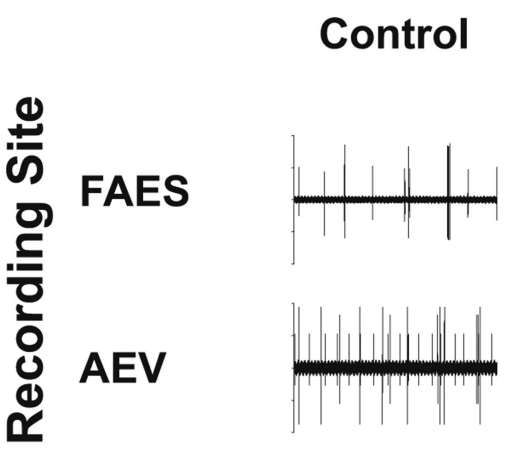

Cooling coil In AEV

\section{Cooling coil active}

Recovery
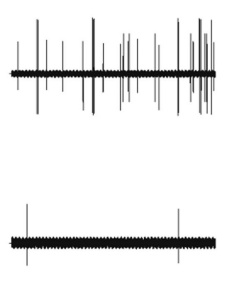

ings were used to guide the placement of the deactivation coils. After identifying the AES subregions via responses to auditory (FAES) or visual (AEV) stimulation, their sulcal walls were gently separated so that a $5 \mathrm{~mm}^{2}$ hollow cryogenic probe, fashioned from loops of 19 gauge stainless-steel hypodermic tubing (inner diameter: $0.8128 \mathrm{~mm}$ ), could be inserted as describe previously (Jiang et al., 2001; Alvarado et al., 2007a). To confirm that coil placement would allow the selective deactivation of these subregions, spontaneous and evoked activity was recorded in each subregion while deactivating the other. As shown in Figure $1 A$, deactivating FAES did not affect neural activity in AEV, and deactivating AEV did not affect neural activity in FAES (Fig. $1 B$ ). After completing the implantation procedures the area was packed with sterile gelfoam and the wound was sutured closed. Postsurgical analgesics (butorphanol tartrate, $0.1-0.4 \mathrm{mg} / \mathrm{kg} / 6 \mathrm{~h}$ ) were administered as needed and antibiotics were given for 7-10 d (ceftriaxone $20 \mathrm{mg} / \mathrm{kg} / \mathrm{bid}$ ). The first recording sessions began $1-5 \mathrm{~d}$ after completing antibiotic treatment.

\section{Recording}

Recording methods were as described previously (Alvarado et al., 2007a,b). Briefly, anesthesia was induced with ketamine hydrochloride $(30 \mathrm{mg} / \mathrm{kg}$ i.m. $)$ and acepromazine maleate (0.3-0.5 mg/kg i.m.) followed by administration of the paralytic pancuronium bromide ( 0.3 $\mathrm{mg} / \mathrm{kg}$ ). Anesthesia and paralysis were maintained by continuous infusion of ketamine hydrochloride $(4-6 \mathrm{mg} / \mathrm{kg})$ and pancuronium bromide $(0.1-0.2 \mathrm{mg} / \mathrm{kg} / \mathrm{h})$ in $5 \%$ dextrose Ringer solution $(3-6 \mathrm{ml} / \mathrm{h})$. Throughout the experiment, the animal was artificially respired. Blood pressure, $\mathrm{CO}_{2}$ (Digital Vital Signs Monitor, VetSpecs VSM7), and body temperature (rectal probe) were continuously monitored. signed to evaluate these two mutually exclusive possibilities by examining multisensory (visual-auditory) and unisensory (visual-visual) integration in visual-auditory SC neurons before, during, and after the reversible deactivation of AEV and FAES, individually and in combination.

\section{Materials and Methods}

All protocols used were approved by the Animal Care and Use Committee of the Wake Forest University School of Medicine, an AAALACaccredited institution, and were conducted in accordance with the Guide for the Care and Use of Laboratory Animals (National Institutes of Health Publication 86-23).

\section{Surgical implantation}

Surgical implantation was performed using procedures similar to those previously described (Jiang et al., 2001; Alvarado et al., 2007a). Briefly, each cat $(n=3)$ was anesthetized with a mixture of ketamine hydrochloride $(30 \mathrm{mg} / \mathrm{kg}$ i.m.) and acepromazine maleate $(0.3-0.5 \mathrm{mg} / \mathrm{kg}$ i.m.), intubated through the mouth, and then maintained with isoflurane (0.5$3 \%)$. The animal was placed in a stereotaxic head-holder and a craniotomy was made over the SC. A stainless-steel recording cylinder was implanted over the craniotomy to permit access to the SC and to serve as an atraumatic head holder during the recording experiments (McHaffie and Stein, 1983).

A second craniotomy was made to expose the AES for implantation of the cryogenic deactivation coils. The dura was opened to expose the cortex and a series of single and multiunit recordings (see below) were conducted to determine the boundaries of FAES and AEV. These record-
Respiratory rate and volume were adjusted to maintain end-tidal $\mathrm{CO}_{2}$ at $\sim 4.0 \%$, and body temperature was maintained at $37-38^{\circ} \mathrm{C}$ using a heating pad.

During the experiment, the head was stabilized using the atraumatic head-holder so that no wounds or pressure points were introduced. Ophthalmic atropine sulfate (1\%) was used to dilate the right pupil, and a contact lens focused the right eye on a tangent screen placed $45 \mathrm{~cm}$ in front of the animal. An opaque lens occluded the left eye. For each recording session, the recording chamber was disinfected and a glassinsulated tungsten electrode (tip diameter: 1-3 $\mu \mathrm{m}$, impedance: 1-3 $\mathrm{M} \Omega$ at $1 \mathrm{kHz}$ ) advanced to the left SC via a hydraulic microdrive. Visualauditory multisensory and unisensory visual neurons were sought using "search" stimuli (see below). Once "isolated," a given neuron's electrical activity was amplified, digitized, displayed on an oscilloscope, and played through an audio monitor. The neuron's modality convergence profile was determined, its receptive fields (RF) mapped and sensory tests were conducted. When tests were completed, the electrode traverse was continued to search for another sensory-responsive neuron. At the end of the session, the electrode was withdrawn and the cylinder disinfected and sealed. The animal was allowed to recover from anesthesia and returned to its home cage upon return of stable respiration and locomotion.

\section{Search stimuli}

Visual. Moving or stationary flashing spots and bars of light were projected onto the tangent screen. These stimuli were continuously presented as the electrode was lowered through the SC. When a visually responsive neuron was located, its RF was mapped on the screen. A visual test stimulus was either an individual bar of light or paired bars of light. 

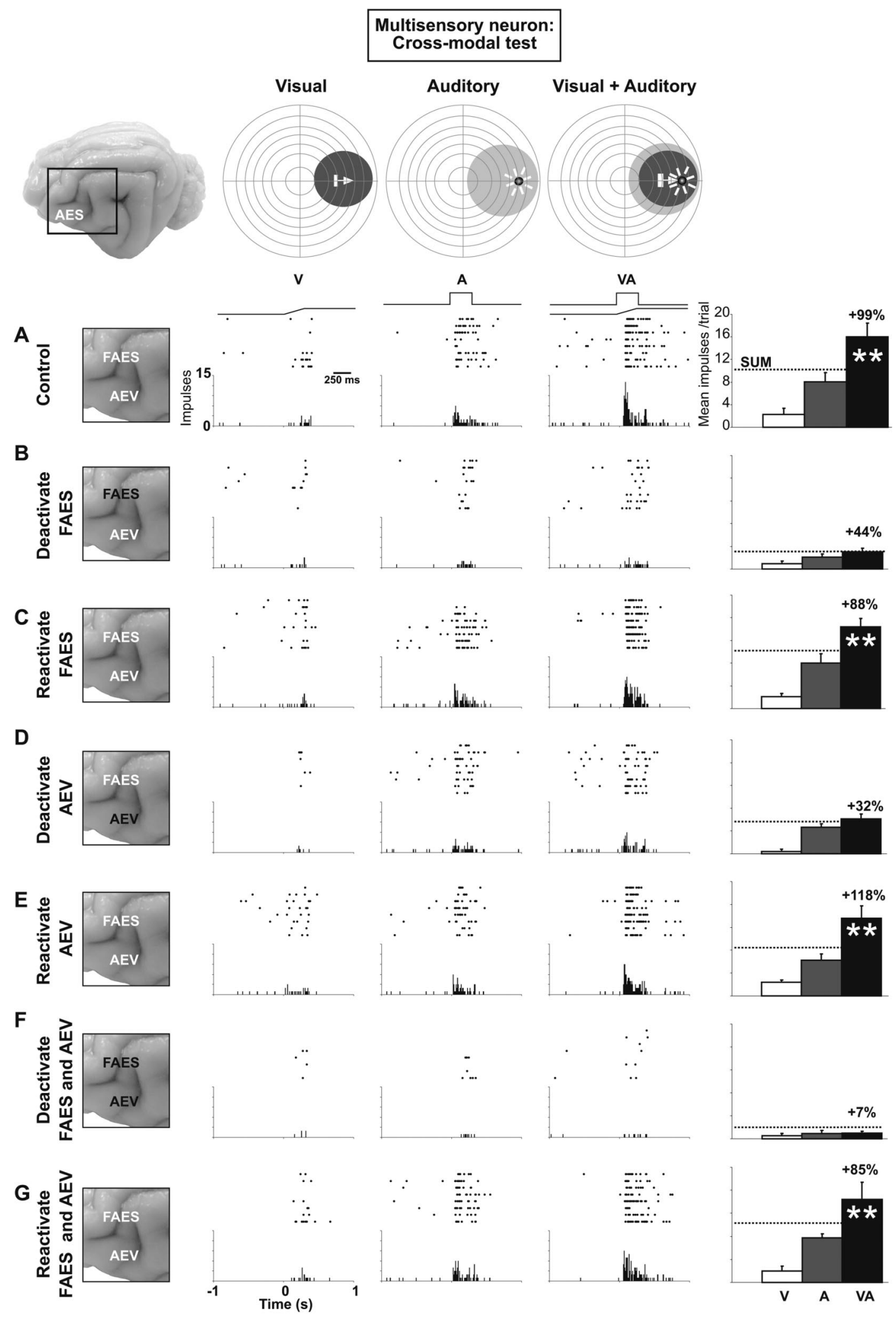

Figure 2. Effect of deactivating AES subregions on SC multisensory integration. Top, Depiction of the visual (dark ovoid) and auditory (light ovoid) RFs of an SC multisensory neuron. Individual deactivation coils were implanted in the auditory (FAES) and visual (AEV) subdivisions of AES cortex under physiological guidance (see Materials and Methods). The visual stimulus was a moving bar of light, and its timing is represented graphically by the ramp $(" V ")$. The auditory stimulus was a broadband noise burst, and its timing is represented by the square pulse (" $A$ "). Below these, the neuron's unisensory and multisensory responses are depicted as rasters, histograms, and summary bar graphs for each stimulus condition tested: control (A), deactivation of FAES $(\boldsymbol{B})$, reactivation $(\boldsymbol{C}$ ) deactivation of AEV $(\boldsymbol{D})$, reactivation $(\boldsymbol{E})$, deactivation of both FAES and AEV $(\boldsymbol{F})$, and final reactivation $(\boldsymbol{G})$. Note that in the control condition $(\boldsymbol{A})$, there was strong multisensory response enhancement (99\%**; "sum" refers to the addition of the two unisensory responses). Deactivation of either FAES $(\boldsymbol{B})$ or AEV (D) eliminated multisensory enhancement and values were no longer significant. Simultaneous deactivation of both subregions $(\boldsymbol{F})$ did not have a significantly greater effect on multisensory integration than did deactivation of either subregion alone. During deactivation, responses to the modality-specific stimuli were significantly reduced in a subregion-specific manner, with auditory and visual responses affected by deactivation of FAES and AEV, respectively $(p<0.05)$. Reactivation of FAES $(\boldsymbol{C}), \operatorname{AEV}(\boldsymbol{E})$, or both $(\boldsymbol{G})$ subregions restored the unisensory and multisensory responses along with multisensory enhancement. ${ }^{* *} p<0.01$. 
Each bar was $0.11-13.0 \mathrm{~cd} / \mathrm{m}^{2}$ against a $0.10 \mathrm{~cd} / \mathrm{m}^{2}$ background, and was projected within the most responsive area of the RF (see Kadunce et al., 1997). The visual stimuli could be flashed on and off at variable rates or moved in any direction across the RF at excursion amplitudes of $1-110^{\circ}$ and speeds of $1-400^{\circ} \%$ s.

Auditory. Broadband noise bursts $(20-20,000 \mathrm{~Hz})$ were delivered through any one of 16 hoop-mounted speakers. The speakers were located $15 \mathrm{~cm}$ in front of the animal and separated by $15^{\circ}$ of arc. The speakers were mounted on a rotating hoop so that elevation could be examined without obstructing the animal's vision or occluding the visual stimulus (see also Meredith and Stein, 1986a,b). Auditory RFs were mapped with broadband noise bursts $10 \mathrm{~dB}$ above threshold for a given neuron and were recorded on standardized maps (Stein and Meredith, 1993). During testing the noise bursts were presented within the most responsive area of the neuron's auditory RF. Burst duration varied from 10 to $50 \mathrm{~ms}$ at intensities of $52-70 \mathrm{~dB}$ SPL against a background SPL of $51.4-52.0 \mathrm{~dB}$.

\section{Cortical deactivation}

Refrigerated water $\left(0^{\circ} \mathrm{C}\right)$ was circulated through the coils (the input and output ends of the coils were exposed above the skin) to deactivate circumscribed regions of cortex, as the deactivated area extends no more than $\sim 2 \mathrm{~mm}$ beyond a given coil (see Jiang et al., 2001). This method has proved to be both effective and reversible in prior studies of corticocollicular influences (Ogasawara et al., 1984; Clemo and Stein, 1986; Jiang et al., 2001, 2002; Jiang and Stein, 2003; Alvarado et al., 2007a). Each subregion deactivation period was $10-15 \mathrm{~min}$, after which cortex was reactivated by circulating warm water $\left(36-38^{\circ} \mathrm{C}\right)$ through the coils for a minimum of $5 \mathrm{~min}$.

\section{General testing paradigm}

Each multisensory (visual-auditory) neuron was tested with three modality-specific ( $\mathrm{V}_{1}$ alone, $\mathrm{V}_{2}$ alone, and $\mathrm{A}$ alone) and two combined $\left(\mathrm{V}_{1}-\mathrm{V}_{2}\right.$ and $\left.\mathrm{V}_{1}-\mathrm{A}\right)$ stimuli at the lowest levels of stimulus effectiveness. Each neuron's dynamic range was approximately determined before testing began. Threshold was determined by successively lowering and raising stimulus intensity until the minimum value that resulted in a reliable response was found (Perrault et al., 2005; Stanford et al., 2005; Alvarado et al., 2007a,b).

Once the appropriate stimulus intensity was chosen, it was used in each of the seven experimental conditions: (1) control, (2) during FAES deactivation, (3) after FAES reactivation, (4) during deactivation of AEV, (5) after reactivation of AEV, (6) during deactivation of FAES and AEV simultaneously, and (7) after FAES and AEV reactivation. For all conditions examined in a given neuron, the same stimulus parameters (effectiveness and duration) were used for individual modality-specific and combined stimuli. Eight repetitions of each stimulus were used and the stimulus conditions were interleaved in pseudorandom manner with $8-20 \mathrm{~s}$ between trials. Although the paired within-modal and crossmodal stimuli were usually presented simultaneously, in some case auditory and visual latencies were different to the extent that a $50 \mathrm{~ms}$ delay ( $\mathrm{V}$ before $\mathrm{A}$ ) was required to ensure a robust multisensory interaction (Meredith et al., 1987). The resulting number of trials for multisensory neurons was 280 ( 8 trials $\times 5$ stimuli $\times 7$ conditions). A similar strategy was followed with unisensory visual neurons, where $V_{1}$ alone; $V_{2}$ alone and $\mathrm{V}_{1}-\mathrm{V}_{2}$ were interleaved in pseudorandom manner for a total of 168 trials ( 8 trials $\times 3$ stimuli $\times 7$ conditions).

\section{Data analysis}

Neural activity was evaluated for each experimental condition, and each neuron served as its own control. Neurons that did not return to their predeactivation state after cortical reactivation were excluded from analysis.

Estimation of mean firing rate. Mean firing frequency across trials was determined for a given stimulus and was based on the number of impulses evoked during a common time window for that neuron. Estimates of spontaneous rate were derived from a $1 \mathrm{~s}$ period preceding stimulus onset for each stimulus condition. Spontaneous rates were normalized to the time window over which driven responses were counted (Jiang et al., 2001; Jiang and Stein, 2003) and stimulus-evoked activity was adjusted by subtracting the spontaneous estimate. Spontaneous rate was generally low in this sample.

Enhancement index. Response enhancement is defined as a statistically significant difference in the number of impulses evoked by the combined stimulus (visual-auditory or visual-visual) compared with that evoked by the most effective modality-specific component stimulus. The relative magnitude of response enhancement is given by the enhancement index according to the following formula (Meredith and Stein, 1983):

$$
\text { Enhancement index }=\left[\left(\mathrm{CR}-\mathrm{MSR}_{\max }\right) /\left(\mathrm{MSR}_{\max }\right)\right] \times 100,
$$

where CR is the response (mean number of impulses/trial) evoked by the combined stimulus (i.e., either visual-auditory or visual-visual) and $\mathrm{MSR}_{\max }$ is the response (mean number of impulses/trial) evoked by the most effective modality-specific stimulus.

Contrast index. A standard contrast index (Motter, 1994; Alvarado et al., 2007a) quantified the effect of cortical deactivation on combined responses according to the following formula:

$$
\text { Contrast index }=(\mathrm{CRD}-\mathrm{CRC}) /(\mathrm{CRD}+\mathrm{CRC}) \text {, }
$$

where CRD is the combined response during deactivation (for FAES, $\mathrm{AEV}$, or both) and CRC is the combined response during the control condition (for either cross-modal or within-modal tests). Contrast values are distributed in the range of -1 to 1 , with zero corresponding to no difference between the control and deactivated responses, positive values corresponding to greater activity following deactivation and negative values corresponding to reduced activity following deactivation.

Differences in control and deactivation response profiles (mean impulses, enhancement indices of the combined responses and contrast indices of the combined responses) were further assessed with standard statistical methods using a significance level of $p<0.05$; $t$ tests, ANOVA, Kruskal-Wallis ANOVA, Friedman ANOVA, and $\chi^{2}$ were used, depending on whether the sample distributions met the assumption of normality based on the Kolmogorov-Smirnov test. All data were analyzed using Statistica for Windows, release 7.0 (StatSoft) and expressed as mean \pm SE.

\section{Nomenclature}

Visual-auditory neurons were sought here as a model with which to explore the effects of AES cortex on multisensory integration. Thus, "multisensory" refers specifically to visual-auditory in this context. The visual modality was used to examine the effect of AES on responses to multiple within-modal cues. Thus, the term "unisensory" refers here specifically to the visual comparator response.

\section{Results}

Seventy-two sensory-responsive neurons in the multisensory layers of the SC (i.e., below stratum opticum) were studied. The sample was drawn from all quadrants of the structure and included 41 multisensory (visual-auditory) and 31 unisensory (visual) neurons.

Response properties and RFs were much the same as have been previously reported (King and Palmer, 1985; Meredith and Stein, 1986a; Peck, 1996; Kadunce et al., 1997; Stein, 1998; Wallace et al., 1998; Jiang et al., 2001; Perrault et al., 2003; Stanford et al., 2005; Alvarado et al., 2007a,b). Visual and auditory representations were topographic and aligned with one another. Thus, the visual and auditory RFs of individual multisensory neurons overlapped one another, and it was common to find that spatially and temporally coincident visual-auditory stimuli elicited responses that were significantly enhanced $(39 / 41,95.12 \%)$. In contrast, it was rare for these same neurons $(2 / 28,7.14 \%)$ or their unisensory visual neighbors $(1 / 31,3.23 \%)$ to respond more vigorously to pairs of visual stimuli than to the most effective of them individually. Indeed, responses to visual combinations were often less vigorous than those to the most effective visual stimuli alone (see also Alvarado et al., 2007a,b). 


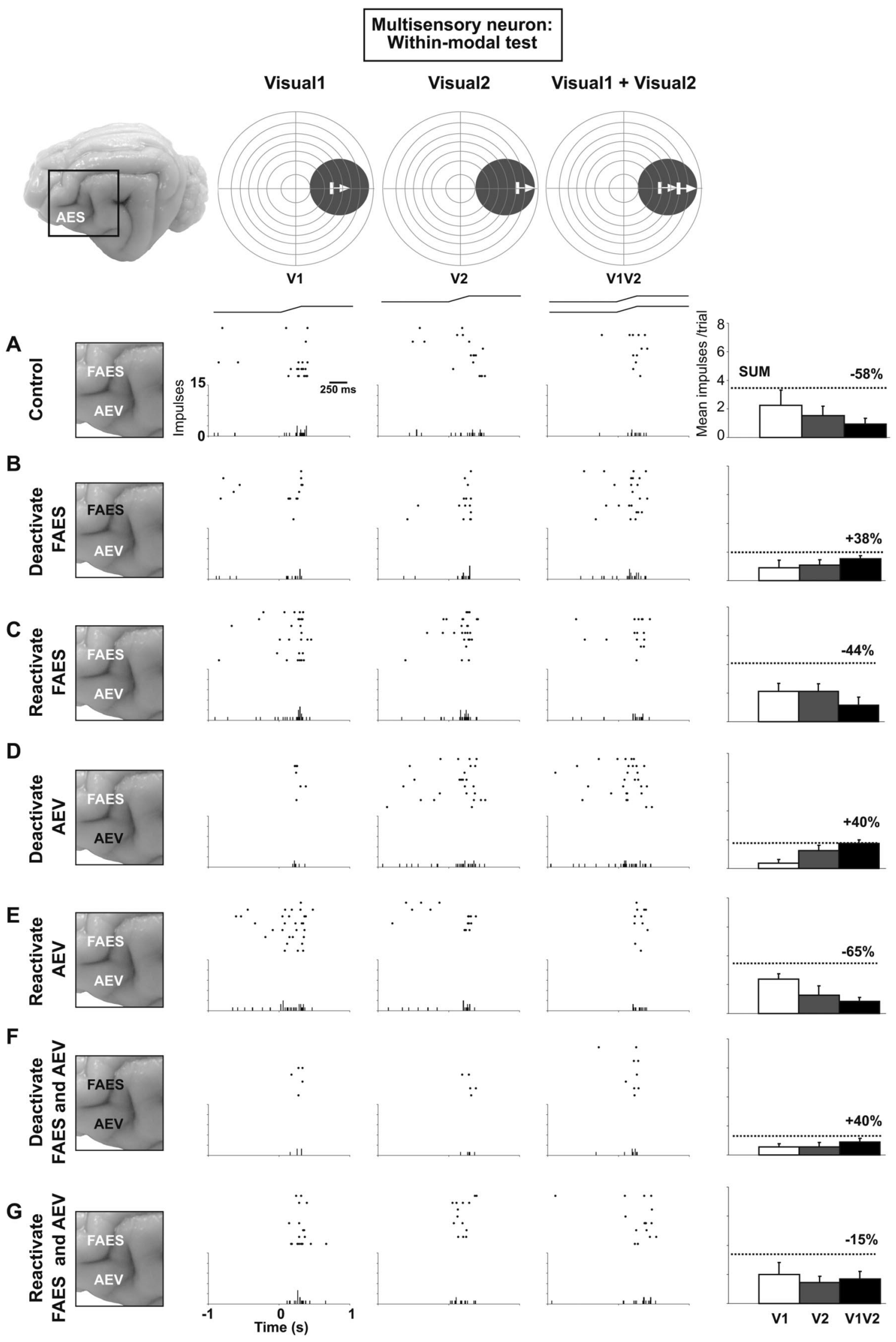

Figure 3. Effect of deactivating AES subregions on unisensory integration in a multisensory SC neuron. The effect of subregion deactivation on unisensory (visual-visual) integration is shown for the same neuron depicted in Figure 2 . In the control condition $(A)$, the within-modal visual stimulus pair produced a response similar in magnitude to that for the more effective of the two visual stimuli presented individually. Thus, no response enhancement was produced by the within-modal stimulus combination. Although deactivation of AEV alone (D) or together with FAES $(\boldsymbol{F})$ diminished the sensory responses, unisensory integration varied little from the control condition. All conventions are the same as in Figure 2. 
Table 1. Frequency and percentage of modality-specific response changes in multisensory and unisensory neurons during cortical deactivation

\begin{tabular}{|c|c|c|c|c|c|}
\hline & \multicolumn{3}{|c|}{ Multisensory neurons } & \multicolumn{2}{|l|}{ Unisensory neurons } \\
\hline & Visual1 $(n=41)$ & Visual $2(n=28)$ & Auditory $(n=41)$ & Visual $1(n=31)$ & Visual $2(n=31)$ \\
\hline \multicolumn{6}{|l|}{ Deactivate FAES } \\
\hline Increase & $1(2.44 \%)$ & $1(3.57 \%)$ & $0(0 \%)$ & $2(6.45 \%)$ & $1(3.23 \%)$ \\
\hline No change & 37 (90.24\%) & $24(85.71 \%)$ & $11(26.83 \%)$ & $28(90.32 \%)$ & $29(93.55 \%)$ \\
\hline Decrease & $3(7.32 \%)$ & $3(10.72 \%)$ & $30(73.17 \%)$ & $1(3.23 \%)$ & $1(3.23 \%)$ \\
\hline \multicolumn{6}{|l|}{ Deactivate AEV } \\
\hline Increase & $0(0 \%)$ & $1(3.57 \%)$ & $1(2.44 \%)$ & $2(6.45 \%)$ & $2(6.45 \%)$ \\
\hline No change & $12(29.27 \%)$ & $8(28.57 \%)$ & $35(85.37 \%)$ & $27(87.10 \%)$ & $29(93.55 \%)$ \\
\hline Decrease & $29(70.73 \%)$ & $19(67.86 \%)$ & $5(12.20 \%)$ & $2(6.45 \%)$ & $0(0 \%)$ \\
\hline \multicolumn{6}{|l|}{ Deactivate both } \\
\hline Increase & $3(7.32 \%)$ & $0(0 \%)$ & $1(2.44 \%)$ & $2(6.45 \%)$ & $1(3.23 \%)$ \\
\hline No change & $10(24.39 \%)$ & $8(28.57 \%)$ & $11(26.83 \%)$ & $26(83.87 \%)$ & $28(90.32 \%)$ \\
\hline Decrease & $28(68.29 \%)$ & $20(71.43 \%)$ & $29(70.73 \%)$ & $3(9.68 \%)$ & $2(6.45 \%)$ \\
\hline
\end{tabular}

Effects of cortical deactivation on multisensory SC neurons Responses to cross-modal stimuli

For most of the multisensory SC neurons examined, the temporary loss of influences from FAES $(78.1 \%, 32 / 41)$, AEV $(73.2 \%$, $30 / 41)$, or both AES subregions $(78.1 \%, 32 / 41)$ had a disproportionate effect on response enhancement. As shown in Figure 2, $B$, $D$, and $F$, cortical deactivation abolished multisensory enhancement but had more modest effects on the responses to either of the modality-specific stimulus components.

In the control condition (Fig. $2 A$ ), the visual-auditory stimulus combination produced a response that was significantly enhanced (99\%) above that to the more effective of the two modality-specific component stimuli. The magnitude of the enhancement obtained here was not unusual among the neurons studied when weakly effective cross-modal stimuli were used, and in some cases, enhancement was considerably greater. In contrast, a marked decrease in multisensory response vigor and, along with it, multisensory enhancement, followed cryogenic blockade of either FAES (Fig. $2 B$ ) or AEV (Fig. 2D) individually or in combination (Fig. $2 F$ ). In each case, multisensory responses were no longer significantly greater than those to the most effective of the modality-specific stimuli.

Although deactivation had the largest impact on the multisensory response, close inspection of Figure 2 reveals that in this case the unisensory responses were also reduced as a consequence of deactivating the matching unisensory AES subregion (Fig. $2 B, D, F$; Table 1). Thus, only deactivating FAES (Fig. $2 B$ ) significantly reduced the auditory response $(p<0.05)$, and only deactivating AEV (Fig. 2D) significantly reduced the visual response $(p<0.05)$. Deactivating both subregions (Fig. $2 F)$ significantly reduced responses to both modality-specific stimuli $(p<0.05)$. Reactivation (warming) of FAES (Fig. 2C), AEV (Fig. 2E) and both subregions (Fig. $2 G$ ) restored the unisensory and multisensory responses along with multisensory enhancement. This was not always the case, and in some examples (Table 1), cortical deactivation produced no change in the unisensory response.

Ordinarily, lowering the magnitude of the most effective unisensory response or both the unisensory responses would be expected to yield even larger multisensory responses to their combination. This relationship between the effectiveness of the component responses and the integrated multisensory response is known as "inverse effectiveness" (Meredith and Stein, 1986) wherein the proportionately largest enhancements are initiated by the least effective modality-specific stimuli. However, during cortical deactivation the principles of multisensory integration are no longer operative (see also Alvarado et al., 2007a). Consequently, it is interesting to note that, despite the very different impact of FAES and AEV deactivation on this neuron's unisensory responses (each was specific to its own modality), the deactivation of each cortical area had nearly equivalent effects on the neuron's multisensory response. Neither AES subregion was able to support multisensory integration/enhancement in the absence of the other. Thus, their combined deactivation was not more detrimental in this regard than was their individual deactivation. In each case multisensory enhancement was lost. Thus, the data suggest the existence of a critical "synergy" in that multisensory integration in this SC neuron that was disrupted by deactivation of only a single subregion and reinstated by reactivation. All neurons were subject to the same test series and exhibited similar results.

\section{Responses to within-modal stimuli}

The impact of removing these cortical influences was highly selective for responses to the cross-modal stimulus. This is illustrated in Figure 3, which demonstrates the absence of equivalent effects on responses to within-modal (visual-visual) stimulus combinations. The simultaneous presentation of two visual stimuli within the RF of this same neuron failed to produce any unisensory response enhancement in the control condition (Fig. $3 A$ ) (see also Alvarado et al., 2007a,b, 2008). In fact, this stimulus combination resulted in a response that was lower than that to either stimulus alone. Also note that as in Figure 2, deactivation of FAES (Fig. 3B) alone had no significant effect on the neuron's visual responses. This occurred only during deactivation of AEV, either alone (Fig. 3D), or in combination with FAES (Fig. $3 F$ ). Furthermore, in no case did cortical deactivation have a disproportionate effect on the combined response as it did when crossmodal stimuli were presented neurons.

\section{Effects of cortical deactivation on unisensory neurons}

To determine whether the influences of FAES and AEV subregions on the unisensory processes were specific to multisensory SC neurons, within-modal tests were also conducted in neighboring unisensory neurons. A representative example is shown in Figure 4. As was the case for similar tests in multisensory neurons (see above), there was essentially no response enhancement resulting from the copresentation of two visual stimuli. In other words, the response initiated by the within-modal stimulus pair was not significantly different from that initiated by either of the individual component stimuli. Among the population examined, within-modal stimulus pairs generally elicited a response that was equal to, or less than, the responses to the component stimuli regardless of whether these tests were conducted in multisensory or unisensory neurons (see also Alvarado et al., 2007a,b, 2008). 


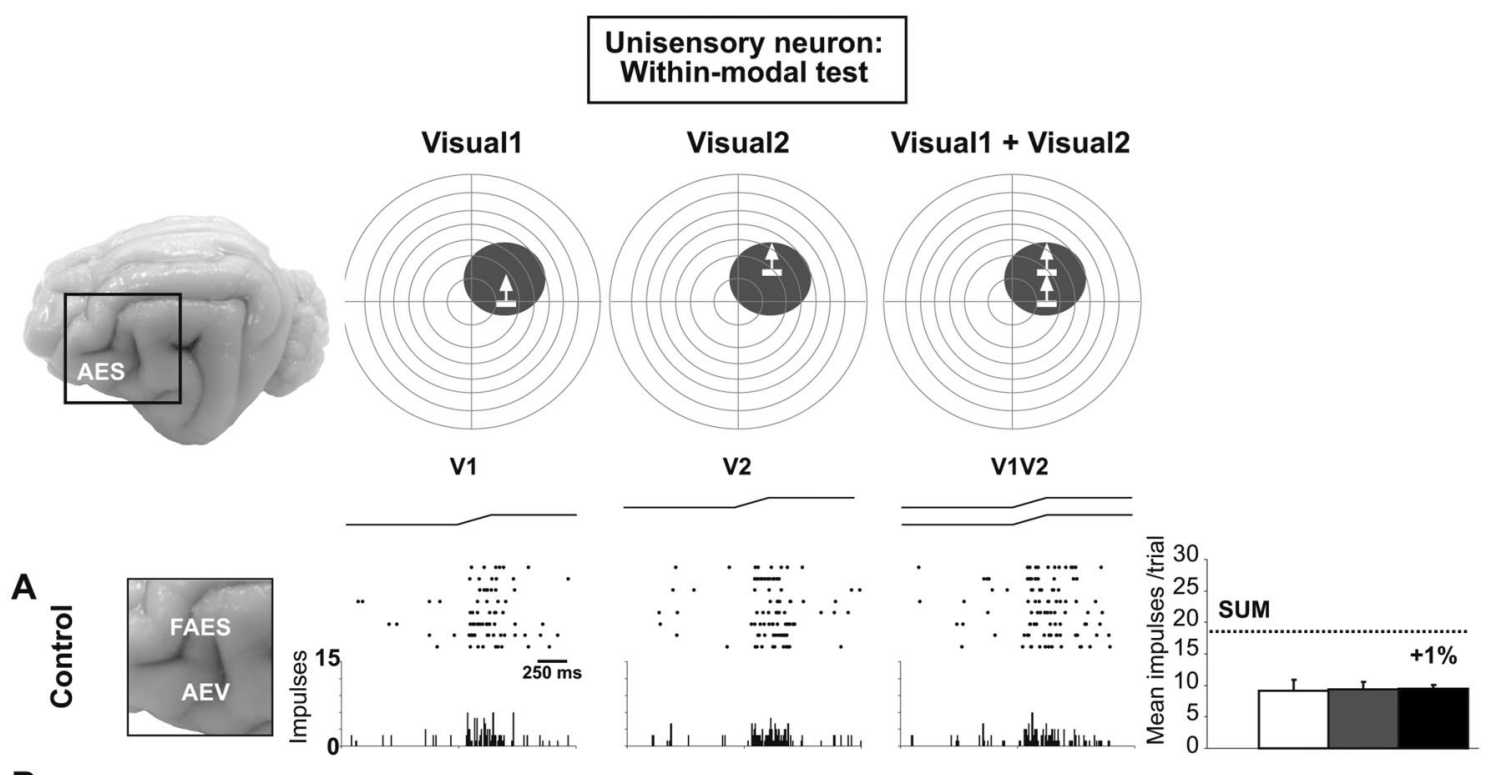

B
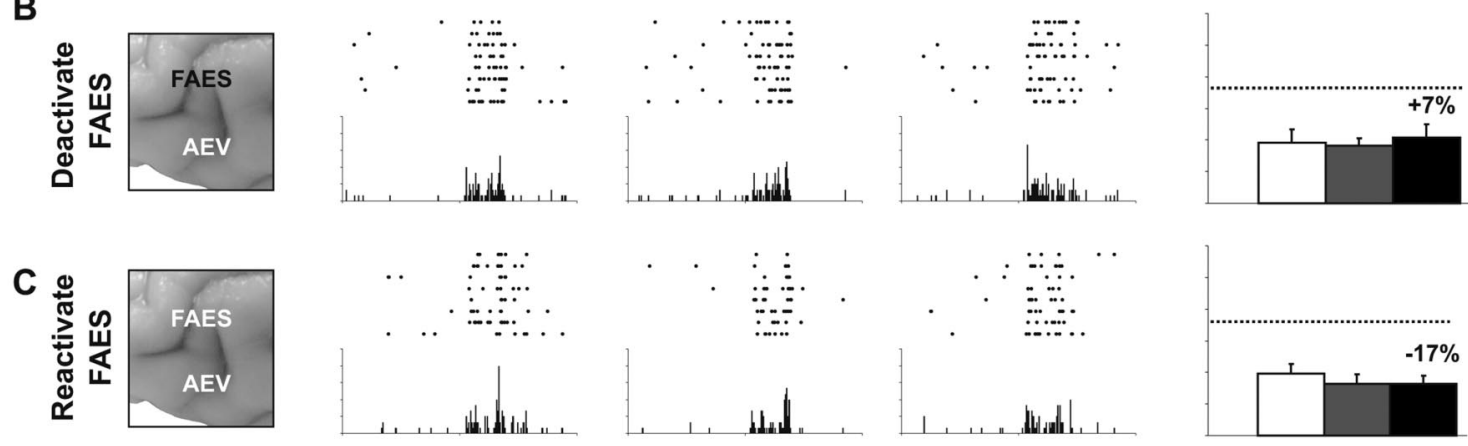

D
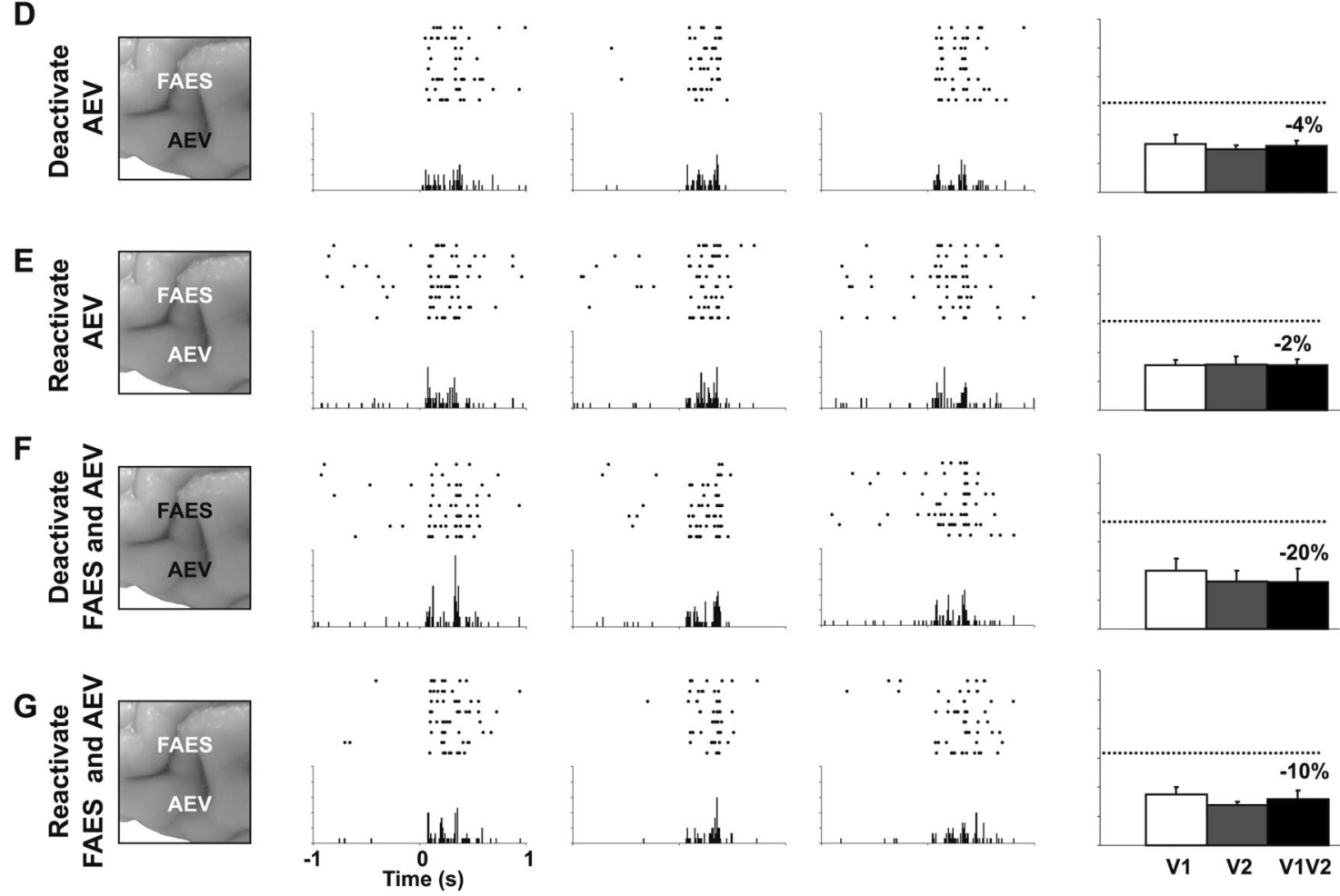

Figure 4. Effect of deactivating AES subregions on unisensory integration in a unisensory SC neuron. The characteristic effect of AES subregion deactivation on unisensory integration (visualvisual) is shown for a unisensory visual SC neuron. As in the multisensory neuron depicted in Figure 3, the within-modal visual stimulus pair failed to produce significant response enhancement (1\%) in the control condition $(\boldsymbol{A})$. Similarly, cortical deactivation of one or both $(\boldsymbol{B}, \boldsymbol{D}, \boldsymbol{F})$ subdivisions did not significantly alter unisensory integration in terms of response enhancement. In contrast to that for the multisensory neuron, deactivation of AEV alone, or in combination with FAES, failed to affect the component visual responses. All conventions are the same as in Figure 2. 


\section{Multisensory neurons: Cross-modal tests}

A

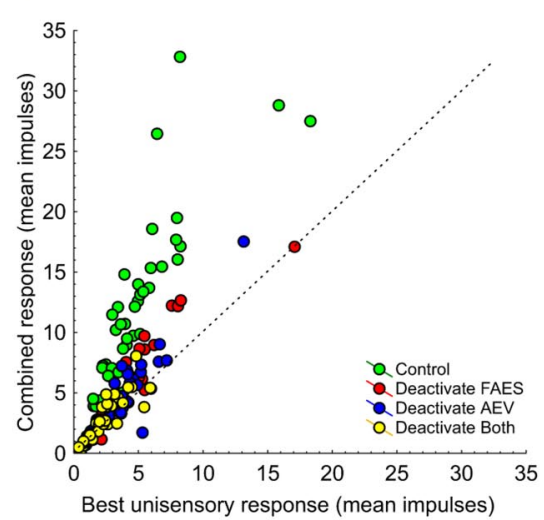

C

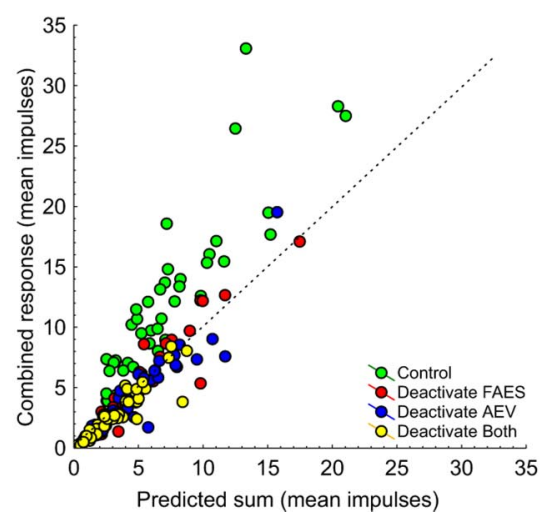

B

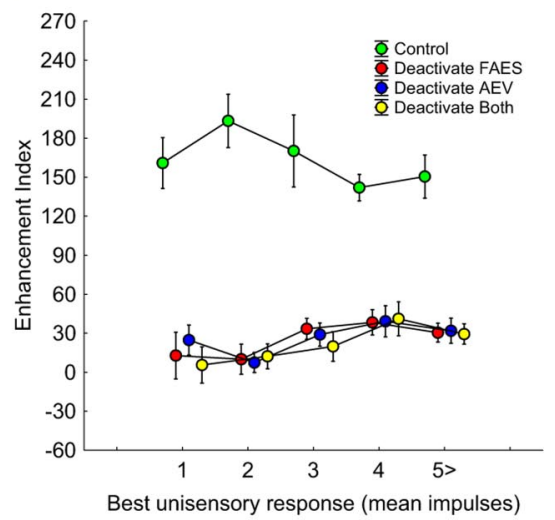

D

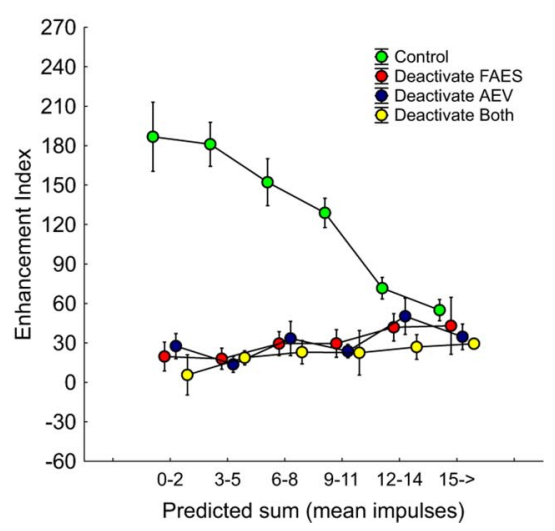

Figure 5. Population effects of AES subregion deactivation on multisensory enhancement. $\boldsymbol{A}$, For each multisensory neuron, the mean response to the cross-modal stimulus combination is plotted against the mean response to the most effective modalityspecific component stimulus for the control (green circles) and cortical deactivation conditions (FAES, red circles; AEV, blue circles; FAES and AEV, yellow circles). In the control condition, (green), multisensory enhancement is indicated by points falling well above the line of unity (the multisensory response exceeds the best unisensory response). During cortical deactivation of FAES, AEV, or both subregions, near complete abolition of enhancement is indicated by points that cluster near the line of unity. $\boldsymbol{B}$, This same effect is seen when comparing the enhancement index to the best unisensory response. During deactivation of either AES subregion, or their combined deactivation, the enhancement index falls toward zero. $\boldsymbol{C}$, The mean response to the cross-modal stimulus combination is plotted against the predicted sum of the responses to the modality-specific component stimuli. Comparison of the control (green) and deactivation (red, blue, yellow) conditions shows that many enhanced multisensory responses exceed the predicted sum (points above line of unity) during the control condition, but become clustered around or below the line of unity during deactivation of one or both AES subregions. $\boldsymbol{D}$, Across all multisensory neurons, the enhancement index is plotted against the sum of the responses to the component unisensory. The control condition (green circles) shows that the magnitude of multisensory enhancement varies inversely with modality-specific stimulus efficacy with very strong enhancement ( $>150 \%)$ for weak stimuli and considerably less $(<60 \%)$ for stronger stimuli. Deactivation of FAES (red circles), AEV (blue circles), or both subregions (yellow circles) renders enhancement minimal across all stimulus efficacies.

However, in contrast to the effects noted in multisensory neurons, cortical deactivation did not demonstrably affect the vigor of the unisensory neuron's response to either component of the within-modal stimulus complex (see Table 1). This finding suggests that neither AES subregion targets unisensory visual neurons in the SC.

\section{Effects of cortical deactivation on the population profiles} Multisensory enhancement

During control trials, significant response enhancement was observed in each of the visual-auditory SC neurons tested, and, as noted above, in almost every case response enhancement was degraded by cortical deactivation. Averaging the effects across the population of neurons recorded yielded a reduction in the mean enhancement index from $163.2 \pm 10.0 \%$ (control) to $27.4 \pm 4.7 \%$ (deactivation of FAES), $26.5 \pm 5.0 \%$ (deactivation of $\mathrm{AEV}$ ), and $21.7 \pm 5.0 \%$ (deactivation of FAES and AEV). Each of these reductions was statistically significant [Kruskal-Wallis $\left.H_{(3,164)}=86.89, p<0.001\right]$. These results are illustrated in Figure $5 A$, which plots the relationship between responses to the combined stimulus (ordinate) and those to the most effective modalityspecific stimulus component (unisensory response-abscissa) for the control (green circles), FAES deactivation (red circles), AEV deactivation (blue circles), and FAES + AEV deactivation (yellow circles) conditions. Before deactivation, strong multisensory enhancement was in evidence as all points fell above the line of equality (green circles). In contrast, deactivation of one or both subregions reduced multisensory responses disproportionately; though generally still above the line of equality, multisensory responses became statistically indistinguishable from the best unisensory response in most cases. This is also evident in Figure $5 B$ where the multisensory enhancement index is plotted as a function of the best unisensory response and is reduced to near zero during the deactivation of FAES, AEV, or both.

The same trend was apparent in Figure 5, $C$ and $D$, which relates the multisensory (combined) response and enhancement index to the predicted sum of the responses to the two modality-specific component stimuli (a composite index of modality-specific stimulus effectiveness). In Figure $5 C$ the control condition all responses to the cross-modal stimuli were above the line of unity, whereas during cortical deactivation of FAES, AEV, and both subregions simultaneously, most points were clustered around or below the line of unity. Figure $5 D$ plots the enhancement index as a function of the predicted sum of responses to the component stimuli. Once again, enhancement values declined steeply with increasing stimulus effectiveness in the control condition. However, during cortical deactivation, the mean enhancement values were uniformly low and did not differ significantly from the prediction at all response levels. Thus, inverse effectiveness, which is typical of multisensory integration, was no longer in evidence. Note that, as shown for the individual example in Figure 2, the deactivation of FAES or AEV produced near equivalent reductions in the multisensory response, reductions that were apparently not different from those in which both AES subregions were deactivated simultaneously.

\section{Unisensory integration}

As shown for the example neuron provided in Figure 3, comparable effects to those described above were not obtained for 
unisensory integration (i.e., within-modal tests) in the population of multisensory neurons examined (Fig. 6). Before and after deactivation in these neurons, the majority of within-modal stimulus pairs failed to yield enhancement, with combined responses approximating the more robust of the two modality specific responses in most cases (Fig. 6A). There was no evidence of response enhancement, with enhancement index values below zero in the control condition and all experimental conditions (Fig. 6B). It is readily apparent from Figure $6, A$ and $B$, that cortical deactivation had little effect on the enhancement index, which shifted from $-17.0 \pm 4.1$ (control) to $-13.3 \pm 3.4$ (FAES deactivation), $-15.6 \pm 3.6$ (AEV deactivation), and $-17.9 \pm 4.4$ (FAES and AEV deactivation).

It is also clear that deactivation of AES subregions individually or in combination had no significant impact on unisensory integration in the population of unisensory neurons examined here. The results look very similar to those obtained in tests of unisensory integration in multisensory neurons. Once again, combined responses in the control condition demonstrated little or no response enhancement (Fig. $7 A, B)$, so that responses in the control and deactivated conditions were very similar. The combined responses were generally well below the predicted sum of the responses to the individual component stimuli so that the enhancement index was well below zero (Fig. 7C,D).

\section{Response magnitude}

The preceding sections showed that deactivation of either of the AES subregions alone virtually abolished multisensory integration in multisensory neurons, but had little impact on unisensory integration in multisensory or unisensory neurons. Thus, with respect to "integration," simultaneous deactivation of both subregions had little or no impact beyond deactivating either subregion alone. However, joint deactivation of the two unisensory subregions did have a slightly greater impact on the overall magnitude of multisensory responses than did deactivation of either subregion alone (Fig. $5 A, C)$. Deactivation of each unisensory cortical subregion alone yielded comparable reductions in the mean of multisensory response magnitude (FAES: $65.4 \pm 2.5 \%$; AEV: $67.9 \pm 2.3 \%$ ), whereas simultaneously deactivating FAES and AEV (79.7 \pm $1.8 \%$ ) had a significantly greater effect than deactivating either one alone (Friedman ANOVA $\chi_{(2,41)}^{2}=41.85, p<0.001$ ). This is illustrated in Figure $8 \mathrm{~A}$, which plots a simple contrast index relating the combined response during cortical deactivation to the combined response in the control condition (see Materials and Methods). Although deactivation of one unisensory subregion (red and blue traces) or both (yellow trace) reduced multisensory response magnitudes as expected (negative contrasts), the greater

\section{Multisensory neurons: Within-modal tests}
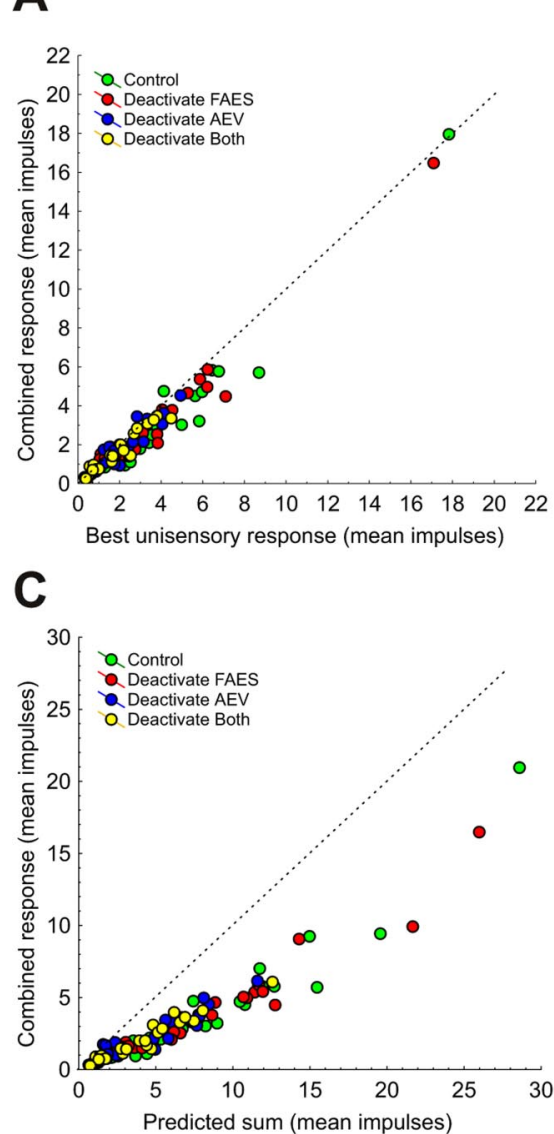

B

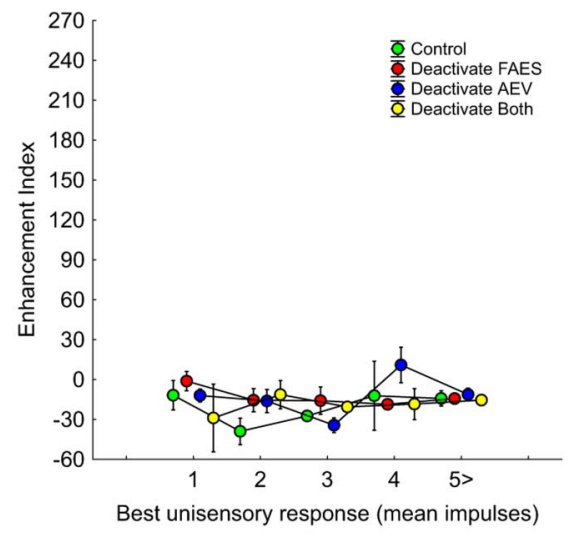

D

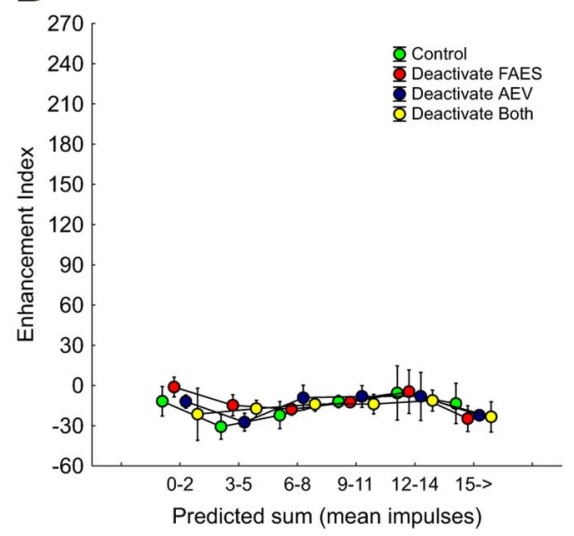

Figure 6. Population effects of AES subregion deactivation on unisensory integration for multisensory neurons. Shown is a the same as in Figure 5.A, A plot of the mean combined response (visual-visual) against the mean best unisensory response shows that within-modal integration fails to yield response enhancement in the control condition (green circles). The points cluster near ity, but some are below the line indicating slight suppression of the response. Cortical deactivation of FAES, AEV, or both subregions had little effect on these combined responses. $\boldsymbol{B}$, Across the population, the enhancement index values hovered produce responses that lie below the line of unity in the control condition (green). These values were unaffected by cortica deactivation of either (or both) AES subdivisions. $\boldsymbol{D}$, The enhancement index was also below zero when plotted against the predicted sum of the two component responses.

reduction produced when deactivating both subregions is evident in the shift toward lower contrast values (yellow trace). The mean value of the contrast index for the deactivation of both subregions was significantly lower than that for deactivating either subregion alone (Friedman ANOVA $\chi_{(2,41)}^{2}=54.58, p<$ $0.001)$.

Figure $8 B$ displays the same analysis for responses to combined within-modal stimuli for these multisensory neurons. As anticipated, there were significant decreases in the response to pairs of visual stimuli only when AEV was deactivated. Thus, the effect of simultaneous deactivation of AEV and FAES was virtually identical to that for AEV alone (Fig. $8 \mathrm{~B}$ ), revealing the specificity of cortical influences. Thus, the mean contrast indices for the deactivation of AEV (alone or simultaneously with FAES) on the visual responses were significantly lower than that for the deactivation of FAES alone (Friedman ANOVA $\chi_{(2,28)}^{2}=42.07$, $p<0.001)$.

In contrast to the effects on multisensory neurons, cortical 


\section{Unisensory neurons: Within-modal tests}

A

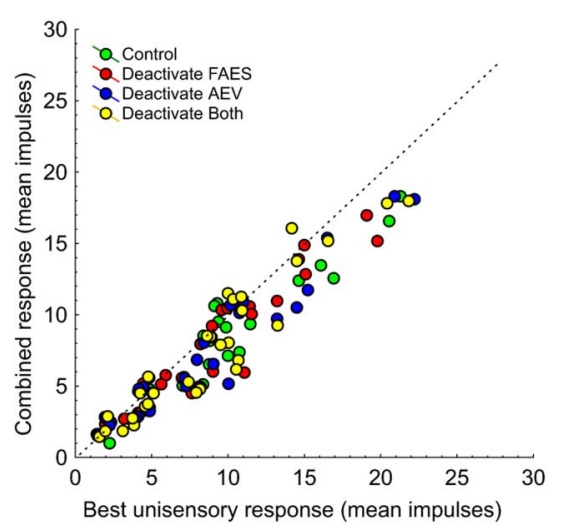

C

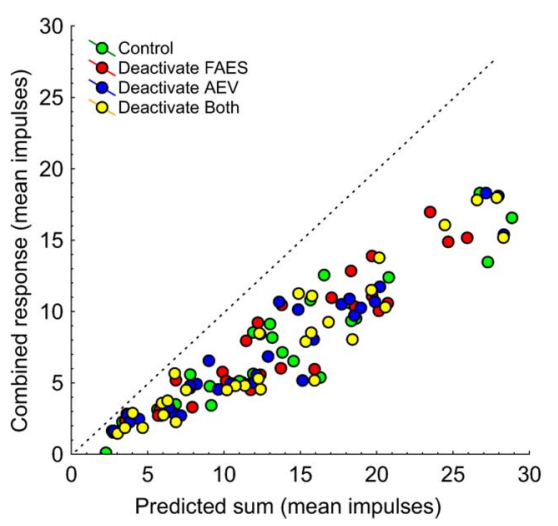

B

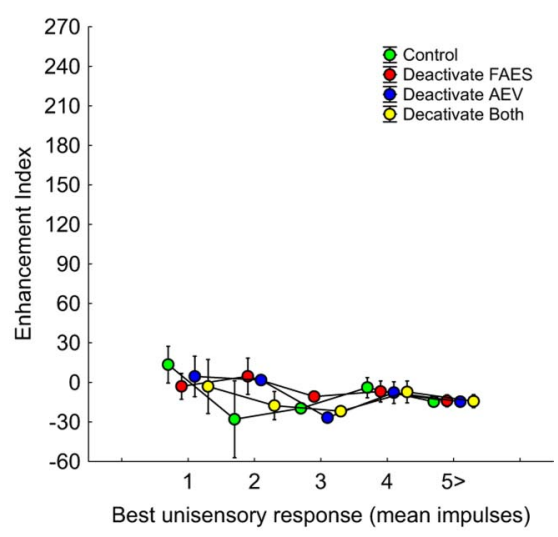

D

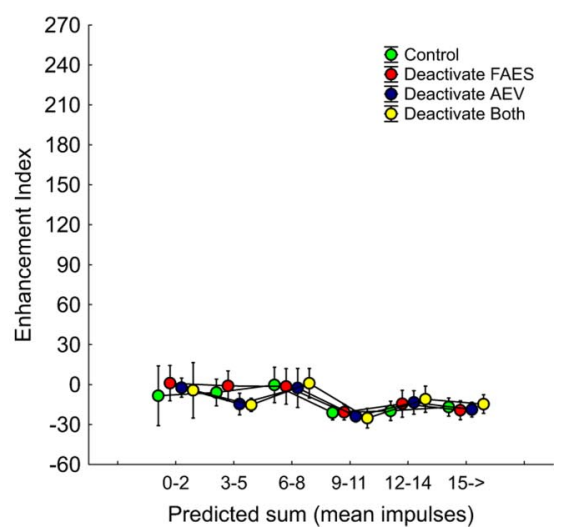

Figure 7. Population effects of AES subregion deactivation on unisensory integration. The same analyses shown in Figure 6 are shown here for the population of unisensory SC neurons and reveal virtually identical trends in each of the analyses. All conventions are the same as in Figure 6.

deactivation had no impact on unisensory visual neurons. Figure $8 C$ shows that cortical subregion deactivation, alone or in combination, did not alter the strength of unisensory (visual) or combined (visual-visual) responses of unisensory neurons (Table 1). All contrast indexes were evenly distributed about zero.

\section{Discussion}

The results demonstrate that converging inputs from the unisensory subdivisions of AES must act in concert to effect multisensory integration in SC target neurons. This inference about the necessity for a synergy among AES projections to the SC is drawn from the observation that for most visual-auditory SC neurons, disrupting the descending influences from either the visual (AEV) or auditory (FAES) subdivision of AES had virtually the same effect as the simultaneous disruption of both: the elimination of visual-auditory multisensory integration. Apparently, neither input alone is sufficient to support this capacity in SC neurons.

Previous studies have shown that the corticocollicular afferents (Stein et al., 1983; McHaffie et al., 1988) critical for multisensory integration arise from unisensory neurons located within three largely unisensory AES subregions (Wallace et al., 1993) composed of visual (AEV), auditory (FAES), and somatosensory (SIV) neurons. Projections from these unisensory regions target multisensory SC neurons with the matching modality-convergence profiles. Thus, visual-auditory SC neurons receive input from the visual (AEV) and auditory (FAES) subdivisions of AES whereas visual-somatosensory and auditorysomatosensory SC neurons receive joint inputs from AEV/SIV and FAES/SIV, respectively.

That the inherent synergy of these corticocollicular projections plays a critical role in determining the responses of SC neurons may seem counterintuitive at first because SC neurons receive a substantial amount of their visual and auditory (and somatosensory) input from non-AES sources (Stein and Meredith, 1993; Edwards et al., 1997; Harting et al., 1997). Furthermore, their unisensory responses retain much of their efficacy even when the entire AES is deactivated (Wallace and Stein, 1994; Jiang et al., 2001; Alvarado et al., 2007a). However, together these results suggest that the AES-SC projection implements a computation that is functionally distinct from that used by SC inputs derived from non-AES sources. Some caution must be exercised here in drawing the conclusion that the observed synergy yielding multisensory integration operates exclusively between the direct influences derived from AES subregions. Deactivation of subregions of AES can also affect the functional integrity of other tectopetal areas. Nevertheless, this interpretation is the most parsimonious to us because, for multisensory behaviors that are SC mediated (Stein et al., 1988, 1989; Jiang et al., 2002; Burnett et al., 2004, 2007), deactivation of other tectopetal areas has, thus far, yielded no apparent impact (Wilkinson et al., 1996). However, this does not obviate the possibility that some more complex neural circuitry involving AES and other tectopetal inputs is engaged in this synergy.

Although there is now a wealth of evidence suggesting a modulatory role for AES influences in SC multisensory integration (Stein, 1998, 2005), little formal consideration has been given to the utility of such a circuit. If one accepts that the SC is engaged in detecting and localizing stimuli, then it might seem odd that its sensory responses would be so dependent on the inputs from an area (AES) with substantially poorer spatial resolution (i.e., larger RFs) than its other sensory afferents (Wallace et al., 1993; Stein and Wallace, 1996).

The most appealing hypothesis, but one that has yet to be tested explicitly in this context, is that these cortical influences confer integrative flexibility such that multisensory integration, and multisensory enhancement in particular, is sensitive to behavioral context. Although the notion that multisensory stimuli adhere to spatial and temporal rules wherein concordant stimuli reinforce and discordant stimuli compete might seem like a functional imperative, it would be maladaptive if these rules were immutable. It is a given that not every instance in which multiple modality-specific stimuli occur coincident in space and time 


\section{A Multisensory neurons: Cross-modal tests}

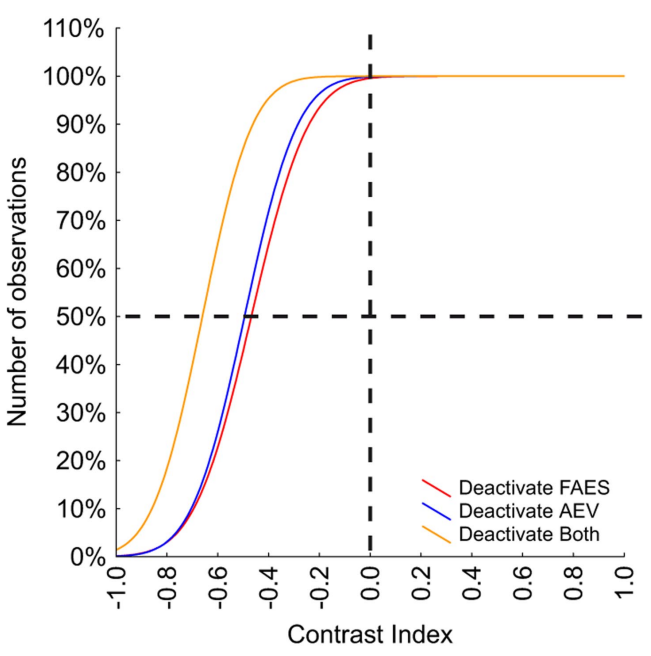

B Multisensory neurons: Within-modal tests

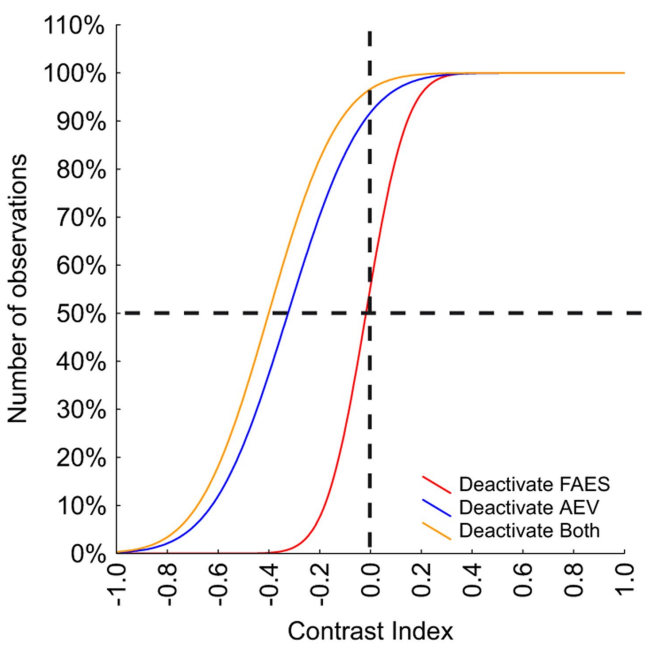

\section{Unisensory neurons: Within-modal tests}

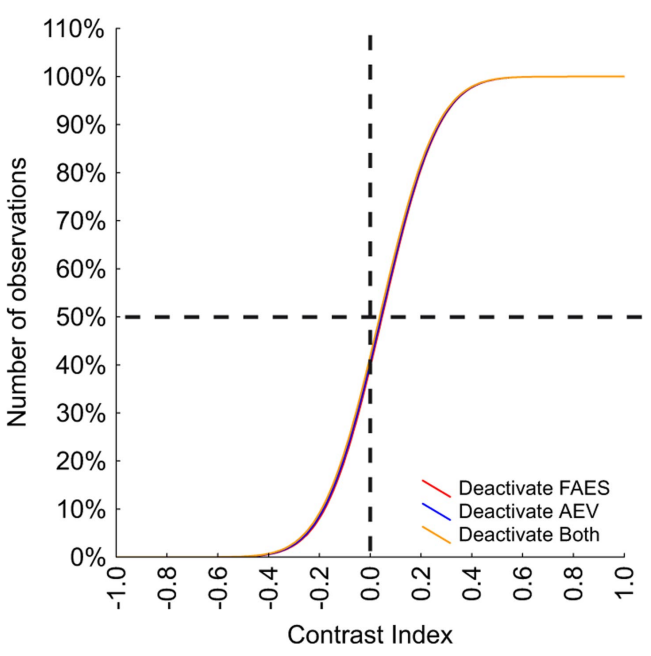

should lead to a speeded orienting response. Though inherently salient, such stimulus combinations could ultimately turn out to be irrelevant, unrewarding, or unreliable (Morgan et al., 2008) and as such, orienting to them would be inconsistent with current behavioral objectives. Consider, for example, that in natural environments cross-modal stimuli might be approximately concordant in space and time but belong to different events. In such a scenario, an optimal system would implement a mechanism that uses other considerations to determine whether or not stimuli belong to the same event before their integration. In addition, such a mechanism could prove very useful if other constraints suggest that certain cross-modal stimulus combinations are irrelevant or unrewarding despite their inherent salience. Thus, a "top-down" mechanism for modulating multisensory integration would seem essential if learned stimulus associations are to inform SC-mediated behavior. Whether or not SC multisensory integration is sensitive to reward contingency and, if so, whether such sensitivity depends on input from AES has yet to be explored.

Also, there is a growing appreciation that much can be gained by viewing multisensory integration within the framework of a Bayesian model. In such a model, the information sources provided by independent sensory channels are fused to yield an improved estimate of an environmental variable, such as stimulus location (Knill and Pouget, 2004; Bresciani et al., 2006; Rowland et al., 2007a; Wozny et al., 2008). In the present context, the magnitude of the SC neuron's response can be interpreted as reflecting the probability that a stimulus is present at a given location (Patton and Anastasio, 2003). This response is the product of both the signal strengths on the individual unisensory input channels representing a particular location in space and the expectation that the modality-specific stimuli will appear at that location. In Bayesian terms, the former are the likelihood distributions and are tied to the physical saliencies (e.g., intensity) of the stimuli, and the latter constitute the prior distributions reflecting the expectation that such a stimulus would occur based on prior experience.

We hypothesize that information carried by unisensory AES inputs represents the experience-based prior distributions that serve to modulate the response to non-AES-derived unisensory inputs. This suggestion has been advanced earlier by Rowland et al. (2007b) and is particularly provocative given the present findings. The prior distribution suggested by Rowland et al. (2007b)

$\leftarrow$

Figure 8. Population effects of subregion deactivation on multisensory and unisensory response magnitudes for multisensory and unisensory neurons. Cumulative density functions are plotted here that relate response magnitudes to combined stimuli for each of the deactivation conditions for multisensory neurons $(\boldsymbol{A}, \boldsymbol{B})$ and unisensory neurons $(\boldsymbol{C})$. The distributions are of a simple contrast index that compares combined response magnitude after deactivation (CRD) to that in the control condition (CRC) according to the following formula: Contrast index = $(C R D-C R C) /(C R D+C R C)$ (see Materials and Methods). $A$, For multisensory neurons, contrast index distributions for cross-modal stimulus combinations are shown for deactivation of FAES (red line), AEV (blue line), and both subdivisions (yellow line). All contrasts are negative indicating that deactivation reduced response magnitude relative to the control condition. Significantly greater reductions in response magnitude were observed for simultaneous deactivation of FAES and AEV (yellow line) than for either alone. $\boldsymbol{B}$, Distributions for within-modal (visualvisual) stimulus combinations for the same multisensory neurons shown in $A$. Consistent with the subregion modality specificity, a reduction in response to visual-visual stimulus combinations magnitude (negative contrasts) was observed only for deactivation conditions that included AEV (blue line, yellow line). C, For unisensory neurons, contrast index distributions for within-modal stimulus combinations are centered near zero illustrating that deactivation of AEV, alone or in combination with FAES (the lines are overlapping), had no impact on the responses to visual stimulus combinations. 
for visual-auditory integration is two-dimensional, with each dimension representing input from either the visual (AEV) or auditory (FAES) subdivisions of AES. Removal of either input in the model results in a unisensory prior distribution which does not influence the other sensory modality, and, consequently, the two sensory modalities remain independent and do not yield integrated multisensory products. Thus, the principal finding of this study, that multisensory integration in the SC reflects a synergistic computation on the inputs derived from AEV and FAES, is consistent with a Bayesian framework in which the AES provides a prior distribution that modulates the activity of multisensory SC neurons.

Currently, the mechanisms by which AES influences SC multisensory integration are poorly understood, although several recent models illustrate how multisensory integration can arise from multiple converging afferents (Anastasio et al., 2000; Patton et al., 2002; Patton and Anastasio, 2003; Deneve and Pouget, 2004; Rowland et al., 2007b; Ursino et al., 2009). One particular challenge has been to discern a biologically plausible mechanism that accounts for the independent effects of cortical deactivation on SC unisensory and multisensory responses. Rowland et al. (2007b) proposed a biologically inspired model in which the descending influences from AES can alter SC multisensory integration without substantially altering the unisensory responses of the same target neurons. Of relevance to the present study, the model requires a synergistic interaction among afferents from multiple unisensory AES subdivisions-in this case AEV and FAES. The essential requirement to effect this interaction is that these converging inputs from AEV and FAES terminate on the same electrotonic compartment of the target SC neuron. Interestingly, there is some anatomical evidence to suggest that at least some of these corticocollicular afferents converge in close spatial proximity on the dendrites or soma of SC neurons (V. FuentesSantamaría, J. C. Alvarado, J. G. McHaffie, and B. E. Stein, unpublished observations).

The evidence suggesting that AES projections provide some unisensory excitatory drive along with modulating multisensory integration (Alvarado et al., 2007a) suggests that they can serve a dual role. It is thus interesting to speculate that the pattern of AES input to the SC might reveal a parallel to the "driver" and "modulatory" input concept that has been proposed for corticothalamic circuits (Sherman and Guillery, 1998, 2002; Guillery and Sherman, 2002; Sherman, 2007). According to this scheme, driver inputs provide large terminals that end mainly on proximal dendrites, whereas modulatory inputs use smaller terminals that end on both proximal and distal dendrites (for review, see Sherman, 2007). Recent evidence indicates that both types of cortical terminals contact multisensory output neurons (Fuentes-Santamaría, Alvarado, McHaffie, and Stein, unpublished observations), but their independent or combined effect on multisensory integration is not yet known.

In contrast to the multisensory enhancement exhibited by SC neurons in response to spatiotemporally concordant crossmodal inputs, they show little or no enhancement to concordant within-modal (visual) stimulus pairs (Alvarado et al., 2007a,b, 2008). Their within-modal responses are also unaffected by AES deactivation. This is unlikely to be coincidental. Tectopetal inputs are derived overwhelmingly from unisensory structures (Edwards et al., 1979; Huerta and Harting, 1984; Stein and Meredith, 1991), or from unisensory neurons in multisensory structures (Wallace et al., 1993). Thus, these inputs are integrated de novo at the SC neuron under the direction of AES. In contrast, two visual stimuli placed in the same RF are most likely to activate partially overlapping sets of ascending and descending afferents and are subject to little SC integration and little AES oversight. The fact that purely unisensory visual neurons appear to receive no input from the AES (Alvarado et al., 2007a) provides further support for the notion that this circuit has evolved for the purpose of multisensory integration.

\section{References}

Alvarado JC, Stanford TR, Vaughan JW, Stein BE (2007a) Cortex mediates multisensory but not unisensory integration in superior colliculus. J Neurosci 27:12775-12786.

Alvarado JC, Vaughan JW, Stanford TR, Stein BE (2007b) Multisensory versus unisensory integration: contrasting modes in the superior colliculus. J Neurophysiol 97:3193-3205.

Alvarado JC, Rowland BA, Stanford TR, Stein BE (2008) A neural network model of multisensory integration also accounts for unisensory integration in superior colliculus. Brain Res 1242:13-23.

Anastasio TJ, Patton PE, Belkacem-Boussaid K (2000) Using Bayes' rule to model multisensory enhancement in the superior colliculus. Neural Comput 12:1165-1187.

Benedek G, Mucke L, Norita M, Albowitz B, Creutzfeldt OD (1988) Anterior ectosylvian visual area (AEV) of the cat: physiological properties. Prog Brain Res 75:245-255.

Bresciani JP, Dammeier F, Ernst MO (2006) Vision and touch are automatically integrated for the perception of sequences of events. J Vis 6:554-564.

Burnett LR, Stein BE, Chaponis D, Wallace MT (2004) Superior colliculus lesions preferentially disrupt multisensory orientation. Neuroscience 124:535-547.

Burnett LR, Stein BE, Perrault TJ Jr, Wallace MT (2007) Excitotoxic lesions of the superior colliculus preferentially impact multisensory neurons and multisensory integration. Exp Brain Res 179:325-338.

Clarey JC, Irvine DR (1986) Auditory response properties of neurons in the anterior ectosylvian sulcus of the cat. Brain Res 386(1-2):12-19.

Clemo HR, Stein BE (1982) Somatosensory cortex: a 'new' somatotopic representation. Brain Res 235:162-168.

Clemo HR, Stein BE (1983) Organization of a fourth somatosensory area of cortex in cat. J Neurophysiol 50:910-925.

Clemo HR, Stein BE (1986) Effects of cooling somatosensory cortex on response properties of tactile cells in the superior colliculus. J Neurophysiol 55:1352-1368.

Deneve S, Pouget A (2004) Bayesian multisensory integration and crossmodal spatial links. J Physiol Paris 98:249-258.

Edwards SB, Ginsburgh CL, Henkel CK, Stein BE (1979) Sources of subcortical projections to the superior colliculus in the cat. J Comp Neurol 184:309-329.

Guillery RW, Sherman SM (2002) Thalamic relay functions and their role in corticocortical communication: generalizations from the visual system. Neuron 33:163-175.

Harting JK, Feig S, Van Lieshout DP (1997) Cortical somatosensory and trigeminal inputs to the cat superior colliculus: light and electron microscopic analyses. J Comp Neurol 388:313-326.

Huerta MF, Harting JK (1984) The mammalian superior colliculus: studies of its morphology and connections. In: Comparative neurology of the optic tectum (Vanegas H, ed), pp 687-773. Plenum, New York.

Jiang H, Lepore F, Ptito M, Guillemot JP (1994) Sensory modality distribution in the anterior ectosylvian cortex (AEC) of cats. Exp Brain Res 97:404-414.

Jiang W, Stein BE (2003) Cortex controls multisensory depression in superior colliculus. J Neurophysiol 90:2123-2135.

Jiang W, Wallace MT, Jiang H, Vaughan JW, Stein BE (2001) Two cortical areas mediate multisensory integration in superior colliculus neurons. J Neurophysiol 85:506-522.

Jiang W, Jiang H, Stein BE (2002) Two corticotectal areas facilitate multisensory orientation behavior. J Cogn Neurosci 14:1240-1255.

Jiang W, Jiang H, Rowland BA, Stein BE (2007) Multisensory orientation behavior is disrupted by neonatal cortical ablation. J Neurophysiol 97:557-562.

Kadunce DC, Vaughan JW, Wallace MT, Benedek G, Stein BE (1997) Mechanisms of within- and cross-modality suppression in the superior colliculus. J Neurophysiol 78:2834-2847.

King AJ, Palmer AR (1985) Integration of visual and auditory information 
in bimodal neurones in the guinea-pig superior colliculus. Exp Brain Res 60:492-500.

Knill DC, Pouget A (2004) The Bayesian brain: the role of uncertainty in neural coding and computation. Trends Neurosci 27:712-719.

McHaffie JG, Stein BE (1983) A chronic headholder minimizing facial obstructions. Brain Res Bull 10:859-860.

McHaffie JG, Kruger L, Clemo HR, Stein BE (1988) Corticothalamic and corticotectal somatosensory projections from the anterior ectosylvian sulcus (SIV cortex) in neonatal cats: an anatomical demonstration with HRP and 3H-leucine. J Comp Neurol 274:115-126.

Meredith MA, Clemo HR (1989) Auditory cortical projection from the anterior ectosylvian sulcus (Field AES) to the superior colliculus in the cat: an anatomical and electrophysiological study. J Comp Neurol 289:687-707.

Meredith MA, Stein BE (1983) Interactions among converging sensory inputs in the superior colliculus. Science 221:389-391.

Meredith MA, Stein BE (1986a) Visual, auditory, and somatosensory convergence on cells in superior colliculus results in multisensory integration. J Neurophysiol 56:640-662.

Meredith MA, Stein BE (1986b) Spatial factors determine the activity of multisensory neurons in cat superior colliculus. Brain Res 365:350-354.

Meredith MA, Nemitz JW, Stein BE (1987) Determinants of multisensory integration in superior colliculus neurons. I. Temporal factors. J Neurosci 7:3215-3229.

Meredith MA, Wallace MT, Stein BE (1992) Visual, auditory and somatosensory convergence in output neurons of the cat superior colliculus: multisensory properties of the tecto-reticulo-spinal projection. Exp Brain Res 88:181-186.

Morgan ML, Deangelis GC, Angelaki DE (2008) Multisensory integration in macaque visual cortex depends on cue reliability. Neuron 59:662-673.

Motter BC (1994) Neural correlates of attentive selection for color or luminance in extrastriate area V4. J Neurosci 14:2178-2189.

Mucke L, Norita M, Benedek G, Creutzfeldt O (1982) Physiologic and anatomic investigation of a visual cortical area situated in the ventral bank of the anterior ectosylvian sulcus of the cat. Exp Brain Res 46:1-11.

Ogasawara K, McHaffie JG, Stein BE (1984) Two visual corticotectal systems in cat. J Neurophysiol 52:1226-1245.

Olson CR, Graybiel AM (1987) Ectosylvian visual area of the cat: location, retinotopic organization, and connections. J Comp Neurol 261:277-294.

Patton PE, Anastasio TJ (2003) Modeling cross-modal enhancement and modality-specific suppression in multisensory neurons. Neural Comput 15:783-810.

Patton P, Belkacem-Boussaid K, Anastasio TJ (2002) Multimodality in the superior colliculus: an information theoretic analysis. Brain Res Cogn Brain Res 14:10-19.

Peck CK (1996) Visual-auditory integration in cat superior colliculus: implications for neuronal control of the orienting response. Prog Brain Res 112:167-177.

Perrault TJ Jr, Vaughan JW, Stein BE, Wallace MT (2003) Neuron-specific response characteristics predict the magnitude of multisensory integration. J Neurophysiol 90:4022-4026.

Perrault TJ Jr, Vaughan JW, Stein BE, Wallace MT (2005) Superior colliculus neurons use distinct operational modes in the integration of multisensory stimuli. J Neurophysiol 93:2575-2586.

Rowland B, Stanford T, Stein B (2007a) A Bayesian model unifies multisen- sory spatial localization with the physiological properties of the superior colliculus. Exp Brain Res 180:153-161.

Rowland BA, Stanford TR, Stein BE (2007b) A model of the neural mechanisms underlying multisensory integration in the superior colliculus. Perception 36:1431-1443.

Sherman SM (2007) The thalamus is more than just a relay. Curr Opin Neurobiol 17:417-422.

Sherman SM, Guillery RW (1998) On the actions that one nerve cell can have on another: distinguishing "drivers" from "modulators". Proc Natl Acad Sci U S A 95:7121-7126.

Sherman SM, Guillery RW (2002) The role of the thalamus in the flow of information to the cortex. Philos Trans R Soc Lond B Biol Sci 357:1695-1708

Stanford TR, Quessy S, Stein BE (2005) Evaluating the operations underlying multisensory integration in the cat superior colliculus. J Neurosci 25:6499-6508.

Stein BE (1998) Neural mechanisms for synthesizing sensory information and producing adaptive behaviors. Exp Brain Res 123:124-135.

Stein BE (2005) The development of a dialogue between cortex and midbrain to integrate multisensory information. Exp Brain Res 166:305-315.

Stein BE, Meredith MA (1991) The functional organization of the superior colliculus. In: Electrophysiology of vision, Vol 4 (Leventhal A, ed), pp 85-110. London, UK: Macmillan.

Stein BE, Meredith MA (1993) The merging of the senses. Cambridge, MA: MIT.

Stein BE, Wallace MT (1996) Comparisons of cross-modality integration in midbrain and cortex. Prog Brain Res 112:289-299.

Stein BE, Spencer RF, Edwards SB (1982) Efferent projections of the neonatal superior colliculus: extraoculomotor-related brain stem structures. Brain Res 239:17-28.

Stein BE, Spencer RF, Edwards SB (1983) Corticotectal and corticothalamic efferent projections of SIV somatosensory cortex in the cat. J Neurophysiol 50:896-909.

Stein BE, Huneycutt WS, Meredith MA (1988) Neurons and behavior: the same rules of multisensory integration apply. Brain Res 448:355-358.

Stein BE, Meredith MA, Huneycutt WS, McDade L (1989) Behavioral indices of multisensory integration: orientation to visual cues is afffected by auditory stimuli. J Cogn Neurosci 1:12-24.

Stein BE, Jiang W, Stanford TR (2004) Multisensory integration in single neurons of the midbrain. In: The handbook of multisensory processes (Calvert GA, Spence C, Stein BE, eds), pp 243-264. Cambridge, MA: MIT.

Ursino M, Cuppini C, Magosso E, Serino A, di Pellegrino G (2009) Multisensory integration in the superior colliculus: a neural network model. J Comput Neurosci 26:55-73.

Wallace MT, Stein BE (1994) Cross-modal synthesis in the midbrain depends on input from cortex. J Neurophysiol 71:429-432.

Wallace MT, Meredith MA, Stein BE (1993) Converging influences from visual, auditory, and somatosensory cortices onto output neurons of the superior colliculus. J Neurophysiol 69:1797-1809.

Wallace MT, Meredith MA, Stein BE (1998) Multisensory integration in the superior colliculus of the alert cat. J Neurophysiol 80:1006-1010.

Wilkinson LK, Meredith MA, Stein BE (1996) The role of anterior ectosylvian cortex in cross-modality orientation and approach behavior. Exp Brain Res 112:1-10.

Wozny DR, Beierholm UR, Shams L (2008) Human trimodal perception follows optimal statistical inference. J Vis 8:24.1-11. 